\title{
High-Resolution Infrared Studies of Perdeutero-Spiropentane, $C_{5} D_{8}$
}

\author{
B.A. Erickson, X. Ju, J.W. Nibler ${ }^{* *}$, C.M. Beaudry, and T.A. Blake ${ }^{\mathrm{a}}$ \\ Department of Chemistry, Oregon State University, Corvallis, OR, 97332-4003 \\ ${ }^{a}$ Pacific Northwest National Laboratory, P.O. Box 999, Mail Stop K8-88, Richland, WA \\ 99352
}

Number of text pages: 20

Number of Tables: 3

Number of Figures: 4

** Corresponding author.

E-mail address: Niblerj@,chem.orst.edu

FAX: +1 5417372062

Keywords: Spiropentane, $\mathrm{C}_{5} \mathrm{H}_{8}$ and $\mathrm{C}_{5} \mathrm{D}_{8}$; High-resolution infrared spectrum;

Rovibrational constants; Molecular structure: DFT study; Anharmonic frequencies 


\begin{abstract}
Perdeutero-spiropentane $\left(\mathrm{C}_{5} \mathrm{D}_{8}\right)$ has been synthesized, and infrared and Raman spectra are reported for the first time. Wavenumber assignments are made for most of the fundamental vibrational states. Gas phase infrared spectra were recorded at a resolution $\left(0.002 \mathrm{~cm}^{-1}\right)$ sufficient to resolve individual rovibrational lines and show evidence of strong Coriolis and/or Fermi resonance interactions for most bands. However a detailed rovibrational analysis of the fundamental $v_{15}\left(b_{2}\right)$ parallel band proved possible, and a fit of more than 1600 lines yielded a band origin of $1053.84465(10) \mathrm{cm}^{-1}$ and ground state constants (in units of $\mathrm{cm}^{-1}$ ): $B_{0}=0.1120700(9), D_{J}=1.51(3) \times 10^{-8}, D_{J K}=3.42(15) \times 10^{-8}$. We note that the $B_{0}$ value is significantly less than a value of $B_{a}=0.1140 \mathrm{~cm}^{-1}$ calculated using structural parameters from an earlier electron diffraction (ED) study, whereas one expects $B_{a}$ to be lower than $B_{0}$ because of thermal averaging over higher vibrational levels. A similar discrepancy was noted in an earlier study of $\mathrm{C}_{5} \mathrm{H}_{8}$ [1]. The structural and spectroscopic results are in good accord with values computed at the anharmonic level using the B3LYP density functional method with a cc-pVTZ basis set.
\end{abstract}




\section{Introduction}

In recent papers we have reported the results of high-resolution infrared studies of several interesting carbon ring compounds of high symmetry, including spiropentane[1,2], propellane [3-5], and bicyclopentane [6,7]. In each case, the molecules are of considerable interest to structural chemists because of their high ring strain and, in the case of propellane, because of the very unusual axial bond. All three molecules are symmetric tops and relatively small but none had previously been examined at a spectroscopic resolution sufficient to discern individual rovibrational transitions. This may be because none of these compounds is available from commercial sources, although synthesis methods for each have been published [8-10]. Using these methods we have prepared small amounts of each compound with sufficient purity to yield excellent high-resolution infrared spectra.

Part of the motivation for studying such molecules comes from an interest in the use of quantum calculations as an aid in the analysis of the complex vibration-rotation spectral patterns one often observes in high-resolution spectroscopy. Such complexity stems from changes in rotational constants with vibrational states and from Fermi resonance and Coriolis couplings between states. For small molecules, useful estimates of the rovibrational parameters accounting for these effects can be obtained from anharmonic potential energy surfaces calculated by quantum programs such as Gaussian [11]. Such parameters enable spectral simulations that are quite helpful in making initial quantum number assignments of rovibrational transitions in complex spectra. Moreover, once accurate molecular parameters are determined from the spectral analysis, their values serve as added tests of the method and basis sets chosen in the quantum calculations. In this manner, for example, it has been determined that the Gaussian 
B3LYP density functional (DFT) method applied to a cc-pVTZ basis set gives quite good rovibrational parameters at a reasonable investment of computer time. In addition, the calculations give helpful information about relative infrared and Raman band intensities that can further aid in vibrational assignments.

Given in our earlier papers $[1,2]$ is a review of the synthesis and previous studies of spiro[2.2]pentane (spiropentane, $\mathrm{C}_{5} \mathrm{H}_{8}$ ):

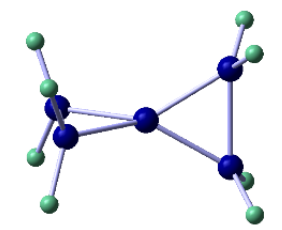
The present work involved a study of the perdeutero form of spiropentane $\left(\mathrm{C}_{5} \mathrm{D}_{8}\right)$, synthesized after the method reported by House et al. [12]. Infrared and Raman spectra were obtained and, aided by the DFT calculations, a confident assignment is made of most vibrational fundamentals. High resolution infrared spectra obtained for gaseous $\mathrm{C}_{5} \mathrm{D}_{8}$ were analyzed to give accurate rovibrational constants for the ground state and the upper state of the $\mathrm{v}_{5}$ transition. Good agreement is found with corresponding parameters obtained from DFT calculations but we note that the experimental $B_{0}$ rotational constant for $\mathrm{C}_{5} \mathrm{D}_{8}$ is inconsistent with a value calculated from the reported electron diffraction structure [13]. A similar inconsistency was seen in our earlier study of $\mathrm{C}_{5} \mathrm{H}_{8}$ [1] and in fact served as partial motivation for the work presented here.

\section{Experimental Details and Results}

\subsection{Synthesis}

The perdeutero-spiropentane (1) was prepared using the method of House et al. [12], with some notable exceptions detailed in Appendix A. The compound was synthesized via four consecutive reactions starting with perdeutero-dibromo ethane (2), as shown in Figure 1. Alkylation of diethyl malonate with $\mathbf{2}$ gave cyclopropyl malonate $\mathbf{3}$. Reduction with lithium aluminum deuteride effectively installed the remaining deuterium 
in diol 4. Activation as the diiodide gave 5. The yields were comparable to those of House et al., except for the last reaction, for which we utilized $\mathrm{Zn}$ metal for the halide extraction and ring-closure step. Our yield for this reduction step was disappointingly low $(<3 \%)$, much less than a value of $20 \%$ reported by House et al. using sodium metal in place of zinc. This poor yield was partially due to the small scale of our reaction amounts, which amplified the losses in the various workup and transfer steps done to purify the final sample after the compound was formed. However the final sample was quite pure, with only about $10 \% \mathrm{C}_{5} \mathrm{D}_{7} \mathrm{H}$ isotopic impurity, and the amount of $\mathrm{C}_{5} \mathrm{D}_{8}$ proved sufficient for the high resolution measurements reported here.

Fig. 1. Reaction sequence for the synthesis of perdeutero-spiropentane $\left(\mathrm{C}_{5} \mathrm{D}_{8}\right)(1)$.
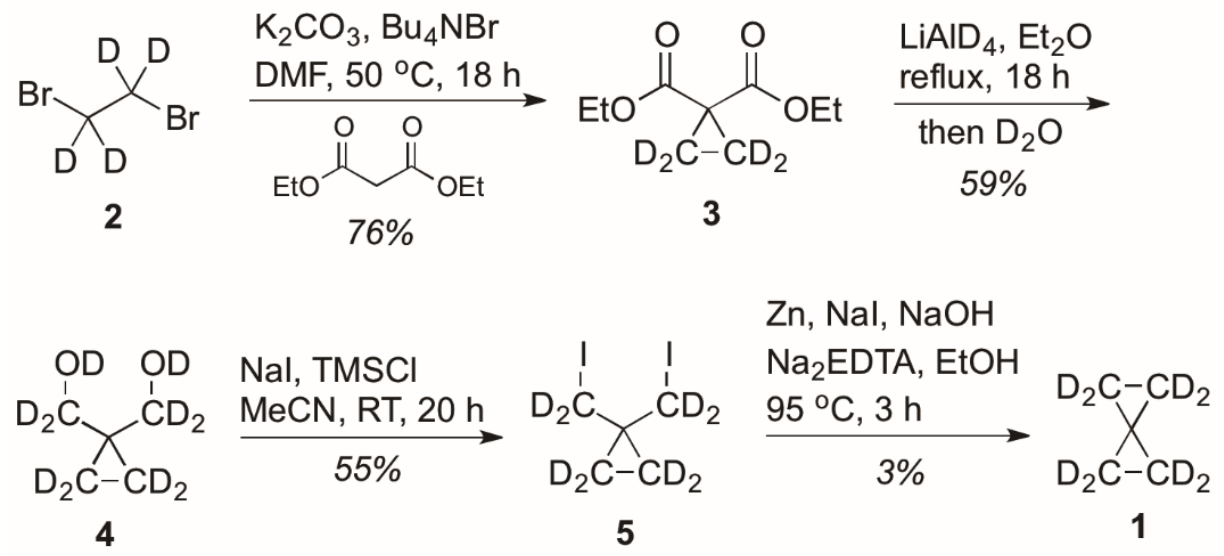

\subsection{Spectroscopy}

In their synthesis paper for $\mathrm{C}_{5} \mathrm{D}_{8}$, House et al. indicated that infrared and Raman spectra would be reported in a subsequent paper; however we have been unable to find any such published report. Accordingly, we show in Figure 2 our infrared spectra for gaseous $\mathrm{C}_{5} \mathrm{D}_{8}$, along with Raman spectra for the liquid phase. The latter were recorded at about $6 \mathrm{~cm}^{-1}$ resolution using a Thermo DXR SmartRaman spectrometer with a 150 $\mathrm{mW} 780 \mathrm{~nm}$ excitation source. 
High-resolution infrared spectra were obtained with the Bruker IFS 125HR Fourier transform spectrometer located at the Pacific Northwest National Laboratory. ${ }^{\dagger}$ In recording the FTIR spectra, a Globar light source, $\mathrm{KBr}$ beamsplitter, and a MCT detector were used. A $20 \mathrm{~cm}$ long gas cell with $\mathrm{KBr}$ windows and temperature-stabilized to $25.0^{\circ} \mathrm{C}$ was filled to about $750 \mathrm{~Pa}$ (5.68 torr) of sample (boiling point $58.7^{\circ} \mathrm{C}$ ) and 4160 interferograms were coadded. Instrument resolution, which is defined as $0.9 /$ (maximum optical path difference), was set to $0.002 \mathrm{~cm}^{-1}$. The averaged interferograms were transformed using a Cooley-Tukey FFT algorithm with four times zero filling, Mertz phase correction, phase resolution set to $1.0 \mathrm{~cm}^{-1}$, and Boxcar apodization. The transition center wavenumber locations were determined by using a second derivative peak-picking algorithm with a nine point Savitsky-Golay smoothing function. No optical filters were used so the spectrum extended from 500 to $4000 \mathrm{~cm}^{-1}$. For the survey scan presented in Figure 2, each data point is the average of 125 points of the high-resolution infrared spectrum. Wavenumber calibration of the various regions was accomplished with an OCS gas reference sample, with wavenumber standards taken from NIST reference tables [14]. The wavenumber corrections were about $0.0002 \mathrm{~cm}^{-1}$, and the absolute wavenumber transition center uncertainty is estimated at $\pm 0.00015 \mathrm{~cm}^{-}$ 1.

\footnotetext{
$\dagger$ Certain commercial equipment, instruments, and materials are identified in the paper to adequately specify the experimental procedure. Such identification does not imply recommendations or endorsements by the Pacific Northwest National Laboratory, nor does it imply that the materials or equipment identified are necessarily the best available for the purpose.
} 
Table 1

Experimental and calculated fundamental frequences $\left(\mathrm{cm}^{-1}\right)$ for spiropentane $\left(\mathrm{C}_{5} \mathrm{D}_{8}\right)$

\begin{tabular}{|c|c|c|c|c|c|c|c|}
\hline \multirow[t]{2}{*}{ Mode } & & \multicolumn{2}{|c|}{ Exp. $^{\mathrm{a}}$} & \multicolumn{4}{|c|}{ Theory (B3LYP/cc-pVTZ) } \\
\hline & & IR & Raman & anharmonic & harmonic & IR int. ${ }^{b}$ & $\begin{array}{c}\text { Raman } \\
\text { int. }\end{array}$ \\
\hline \multirow[t]{5}{*}{$a_{1}$} & $v_{1}$ & $\ldots$ & 2206 & 2202 & 2265 & $\ldots$ & 209.0 \\
\hline & $v_{2}$ & $\ldots$ & 1163 & 1164 & 1191 & $\ldots$ & 12.9 \\
\hline & $v_{3}$ & $\ldots$ & 918 & 925 & 947 & $\ldots$ & 20.2 \\
\hline & $v_{4}$ & $\ldots$ & 798 & 807 & 824 & $\ldots$ & 9.5 \\
\hline & $v_{5}$ & $\ldots$ & 530 & 520 & 533 & $\ldots$ & 11.2 \\
\hline \multirow[t]{3}{*}{$a_{2}$} & $v_{6}$ & $\ldots$ & $\ldots$ & 2294 & 2374 & $\ldots$ & $\ldots$ \\
\hline & $v_{7}$ & $\ldots$ & $\cdots$ & 892 & 908 & $\ldots$ & $\ldots$ \\
\hline & $v_{8}$ & $\ldots$ & $\ldots$ & 609 & 616 & $\ldots$ & $\ldots$ \\
\hline \multirow[t]{4}{*}{$b_{1}$} & $v_{9}$ & $\ldots$ & 2312 & 2300 & 2378 & $\ldots$ & 94.6 \\
\hline & $v_{10}$ & $\ldots$ & & 939 & 954 & $\ldots$ & 4.3 \\
\hline & $v_{11}$ & $\ldots$ & & 733 & 749 & $\ldots$ & 0.5 \\
\hline & $v_{12}$ & $\ldots$ & & 236 & 237 & $\ldots$ & 0.6 \\
\hline \multirow[t]{5}{*}{$b_{2}$} & $v_{13}$ & 2190 & & 2189 & 2269 & 17.7 & 4.8 \\
\hline & $v_{14}$ & & & 1485 & 1529 & 0.1 & 0.1 \\
\hline & $v_{15}$ & 1054 & & 1051 & 1079 & 1.3 & 0.6 \\
\hline & $v_{16}$ & 847 & 840 & 845 & 870 & 4.6 & 10.7 \\
\hline & $v_{17}$ & 724 & & 725 & 735 & 16.4 & 1.3 \\
\hline \multirow[t]{8}{*}{$e$} & $v_{18}$ & 2321 & 2327 & 2300 & 2380 & 26.7 & 60.0 \\
\hline & $v_{19}$ & 2190 & 2172 & 2174 & 2250 & 23.8 & 38.1 \\
\hline & $v_{20}$ & 1081 & & 1074 & 1101 & 10.1 & 0.2 \\
\hline & $v_{21}$ & 1032 & 1026 & 1029 & 1050 & 1.6 & 10.4 \\
\hline & $v_{22}$ & & 861 & 874 & 891 & 0.3 & 5.4 \\
\hline & $v_{23}$ & & 719 & 719 & 731 & 6.8 & 15.3 \\
\hline & $v_{24}$ & & 554 & 560 & 563 & 0.5 & 2.7 \\
\hline & $v_{25}$ & & & 264 & 263 & 0.3 & 0.2 \\
\hline
\end{tabular}

${ }^{\mathrm{a}}$ Infrared values are for the gas phase, Raman values for the liquid phase. 
${ }^{\mathrm{b}}$ Infrared and Raman intensities are harmonic values.

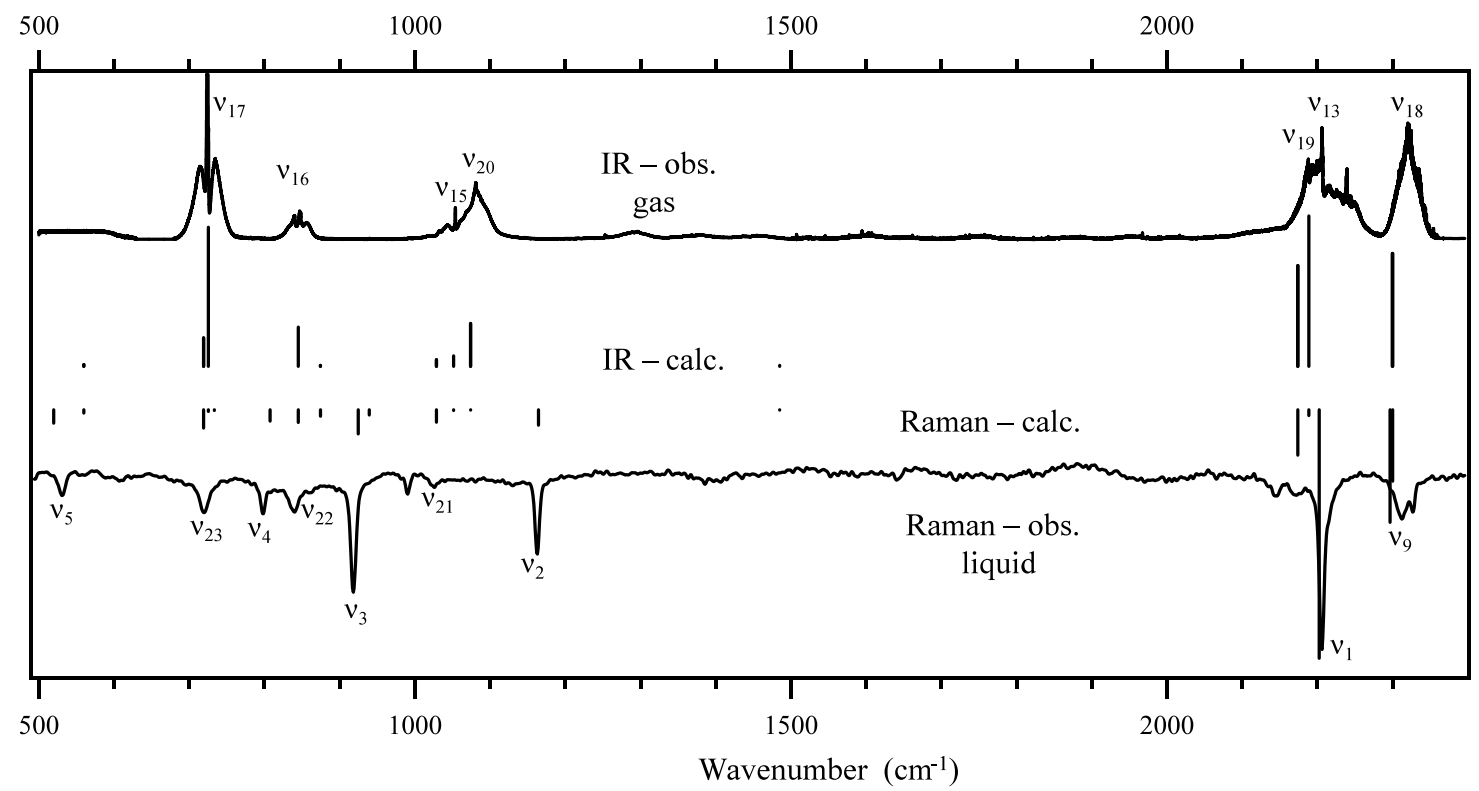

Fig. 2. Infrared and Raman spectra of perdeutero-spiropentane $\left(\mathrm{C}_{5} \mathrm{D}_{8}\right)$. For display purposes, intense features due to $\mathrm{CO}_{2}$ and $\mathrm{H}_{2} \mathrm{O}$ were deleted from the spectra. Assignments of most of the fundamental modes are indicated in the spectrum. Vertical sticks indicate the positions calculated at the anharmonic level using Gaussian (B3LYP/cc-pVTZ), with stick heights representing relative intensities computed at the harmonic level.

\subsection{Vibrational Assignments}

Spiropentane has the symmetry of the $D_{2 \mathrm{~d}}$ point group, and the representation of the $3 \mathrm{~N}-6$ normal modes of vibration is

$$
\Gamma_{\mathrm{vib}}=5 a_{1}(\mathrm{R})+3 a_{2}+4 b_{1}(\mathrm{R})+5 b_{2}(\mathrm{IR}, \mathrm{R})+8 e(\mathrm{IR}, \mathrm{R})
$$

The infrared and Raman activities for the fundamental transitions are shown in parentheses. Table 1 lists fundamental frequencies assigned in this work, along with the harmonic and anharmonic values calculated by us using Gaussian 09 (B3LYP method with a cc-PVTZ basis). The harmonic predictions of relative infrared and Raman intensities are also shown for all fundamentals and are indicated as stick heights in Fig. 2. 
The five $a_{1}$ modes were prominent in the Raman spectrum and match closely the calculated anharmonic values. The $a_{2}$ modes are unassigned since they are inactive in both infrared absorption and Raman scattering processes. Of the four Raman active $b_{1}$ modes, only $v_{9}$ is assigned, a $\mathrm{CD}_{2}$ antisymmetric stretch at $2312 \mathrm{~cm}^{-1}$. The five $b_{2}$ fundamental bands are parallel transitions with sharp $Q$-branches that are relatively obvious in the infrared spectrum for four modes. A detailed analysis of the $v_{15} b_{2}$ mode, described below, gave a very accurate value of the rovibrational parameters for this fundamental. The fifth $b_{2}$ fundamental, a $\mathrm{CD}_{2}$ bending mode, $v_{14}$, is predicted to have very low infrared and Raman intensity and could not be detected.

The band centers of the eight perpendicular $e$ bands are less well-defined but tentative assignments are made for seven of these, based on comparisons with the frequencies and intensities predicted by the Gaussian calculations. The normal modes obtained in these calculations predict the symmetric $\mathrm{CD}_{2}$ stretching modes $\left(v_{1}, v_{13}, v_{19}\right)$ to be about $2200 \mathrm{~cm}^{-1}$ while the antisymmetric stretches $\left(v_{6}, v_{9}, v_{18}\right)$ are higher, near $2300 \mathrm{~cm}^{-}$ 1. Because of band overlaps and Coriolis couplings between $b_{2}$ and $e$ states, most gas phase band centers have an uncertainty of about 2 to $5 \mathrm{~cm}^{-1}$, and a similar uncertainty applies to the Raman values, which are for the liquid phase. Some unassigned features in the two spectra are believed to be combination bands or $\mathrm{C}_{5} \mathrm{D}_{7} \mathrm{H}$ features. (DFT calculations for $\mathrm{C}_{5} \mathrm{D}_{7} \mathrm{H}$ predict that the two most intense IR features are at 3016 and $733 \mathrm{~cm}^{-1}$, regions that do not interfere with the analysis presented here.) Overall, the agreement with frequencies and intensities predicted by the DFT calculations is considered quite good.

\section{Rovibrational Band Analyses}

\subsection{Rovibrational level expressions}


Spiropentane is a prolate symmetric top molecule with principal moments of inertia $I_{A}<I_{B}=I_{C}$. The rotational constants are then given as $A>B$ in which $A=$ $h /\left(8 \pi^{2} c I_{A}\right)$, and $B=h /\left(8 \pi^{2} c I_{B}\right)$, where $h$ is Planck's constant and $c$ is the speed of light in vacuum. The physical constants are chosen such that the rotational constants are expressed in units of $\mathrm{cm}^{-1}$.

The energy (in units of $\mathrm{cm}^{-1}$ ) of a given vibrational state, $\mathrm{v}$, is given by the term value expression

$$
E_{v}=G(v, l)+F_{v}(J, K, l)
$$

where $G(v, l)$ is the vibrational term, and $F_{v}(J, K, l)$ is the rotational term, which may be divided into two parts

$$
F_{V}(J, K, l)=F_{v}(J, K)+F_{v_{\perp}}(J, k, l)
$$

The contracted subscript $v$ is used to represent both quantum number and mode number of a vibrational state. For the ground state $v_{1}=v_{2}=v_{3}=\ldots=0$. The zero energy is defined as the $J=K=0$ level of the ground state i.e., $v_{0}=G(v, l)-G(0,0)$. For this and other states with vibrational angular momentum quantum number $l=0$, the second term of Equation 2 is dropped, and $F_{v}(J, K)$ is given by

$$
\begin{aligned}
F_{v}(J, K) & =B_{v} J(J+1)+\left(A_{v}-B_{v}\right) K^{2}-D_{v J} J^{2}(\mathrm{~J}+1)^{2}-D_{v J K} J(J+1) K^{2}-D_{v K} K^{4} \\
& +H_{v J} J^{3}(J+1)^{3}+H_{v J K} J^{2}(J+1)^{2} K^{2}+H_{v K J} J(J+1) K^{4}+H_{v K} K^{6} \\
& \pm \delta_{2 K \mathrm{v} 2}[J(J+1)][J(J+1)-2] .
\end{aligned}
$$

In the above expressions $J$ is the total angular momentum quantum number (exclusive of nuclear spin), $K$ is the quantum number of the projection of the vector $J$ onto the principal $C_{2}$ symmetry axis, $k$ is that of the signed projection of $J$ onto the symmetry axis, so that $K$ $=|k|$. The $D$ and $H$ terms are quartic and sextic corrections for centrifugal distortion and the last ${ }_{\mathrm{v} 2}$ term in Eq. (3) accounts for the centrifugal rotational $K$-type splitting of the $k$ 
$=2$ levels into a $B_{1}, B_{2}$ pair for the $A_{1}$ ground vibrational state or, for a $B_{2}$ vibrational state, into a $A_{1}, A_{2}$ pair. The pattern of levels predicted in the case of a parallel band such as $\mathrm{M}_{5}$ is shown in Figure 3. The nuclear spin weights $\mathrm{g}_{\mathrm{ns}}$ indicated in this figure for the ground levels are 1701 for $K$-even levels of vibronic symmetry $A_{1}$ or $B_{1}$, and 1620 for $K$ even levels of vibronic symmetry $A_{2}$ or $B_{2}$. For $D_{2 \mathrm{~d}}$ molecules, the allowed infrared transitions between rovibrational levels are $A_{1} \leftrightarrow B_{1}, A_{2} \leftrightarrow B_{2}$, and $E \leftrightarrow E[15]$. 


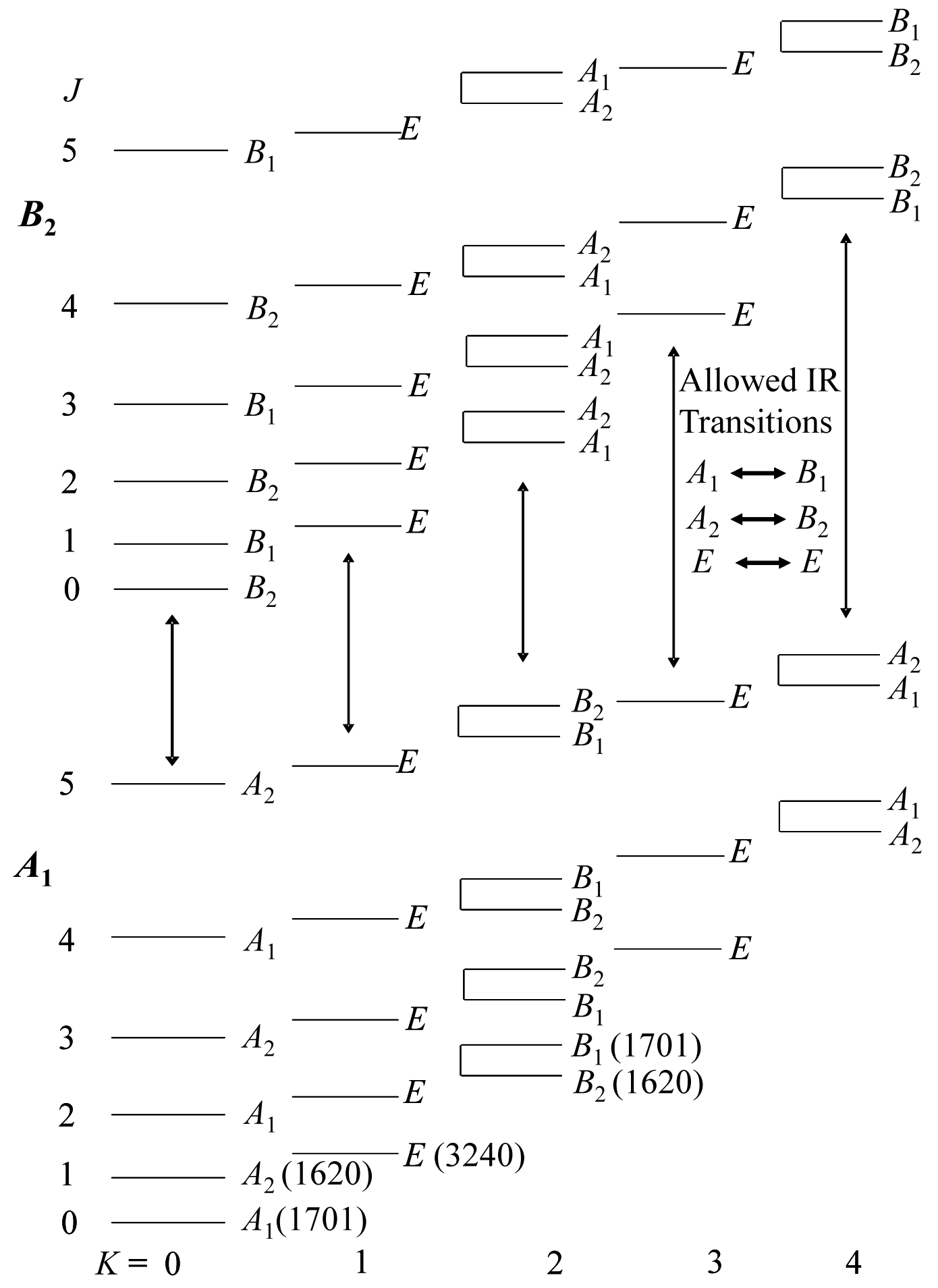

Figure 3. Diagram for the allowed transitions between $A_{1}$ and $B_{2}$ vibrational states for the $\mathrm{M}_{5}$ band of perdeutero-spiropentane, $\mathrm{C}_{5} \mathrm{D}_{8}$. 


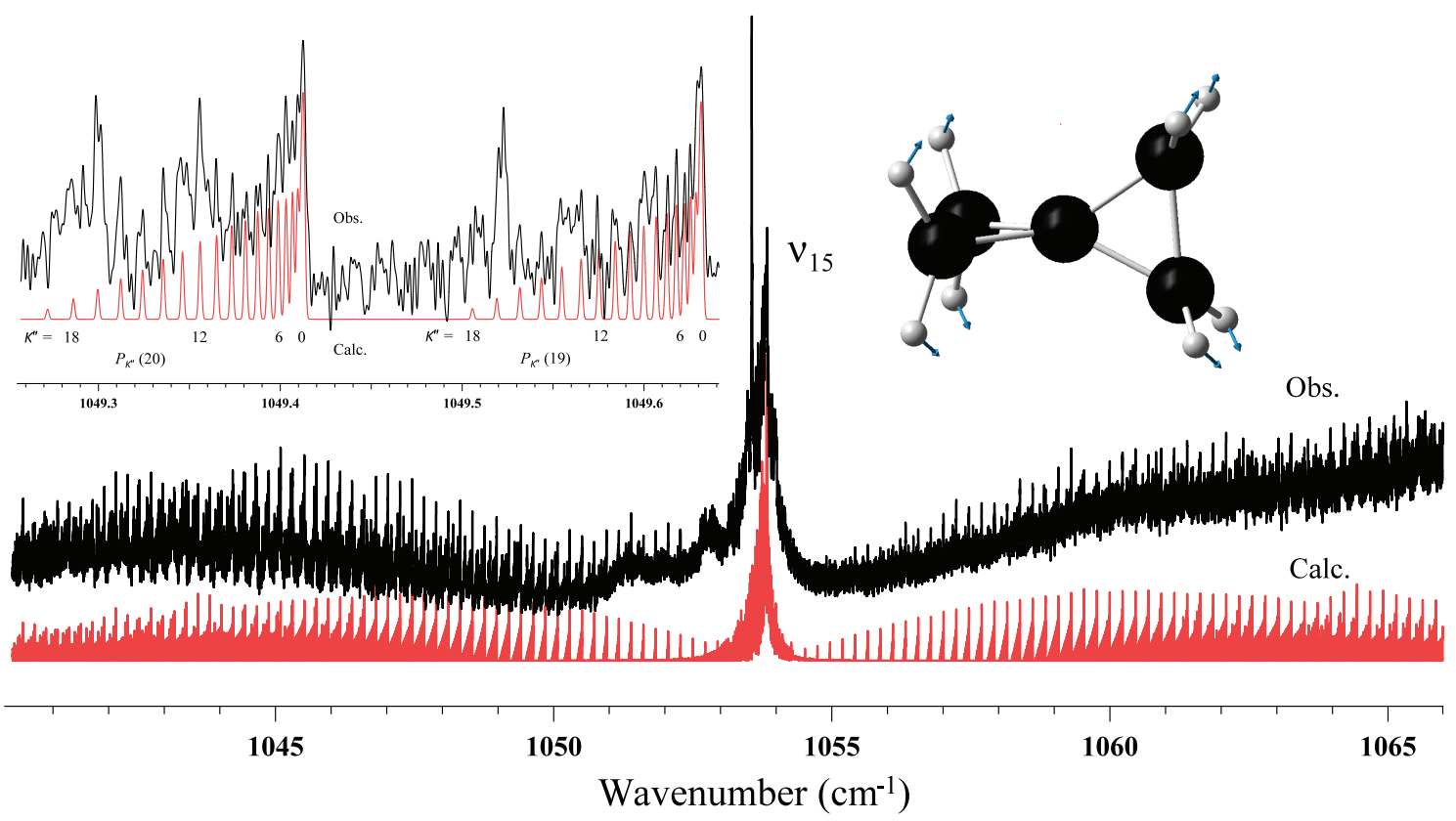

Figure 4. $\cup_{5}\left(b_{2}\right)$ parallel band of perdeutero-spiropentane. The inset to the left shows the $K^{\prime \prime}$-structure of the $J^{\prime \prime}=20$ and $19 P$-lines. Some of the extra features, as well as the sharp $Q$-feature on the left side of the $Q$-branch are attributed to hot bands, such as ( $v_{5}$ $\left.+v_{5}\right)-v_{25}$. The rising baseline in the $R$-branch is due to overlap with a nearby $v_{2}(e)$ perpendicular band at $1081 \mathrm{~cm}^{-1}$.

\subsection{The $v_{15} 1054 \mathrm{~cm}^{-1}$ parallel band}

The $v_{15}$ band, seen in Figure 4, shows the characteristic $P-Q-R$ shape of a parallel band, and the right hand edge of the $Q$-branch defines the band origin, making the $J$ assignment of the $P$ - and $R$-branch lines relatively straight-forward. The left inset of Figure 4 shows the individual resolved $K$-transitions of the $J^{\prime \prime}=19$ and $20 K$-stacks of the $P$-branch recorded in the present work. The nuclear spin statistical weights $\mathrm{g}_{\mathrm{ns}}$ in the ground vibrational state are shown in Figure 3 and, for the special case $K^{\prime \prime}=0$, the $J$-even lines have a weight of 1701 while the $J$-odd lines are weaker, with weight 1620 . In contrast to the case for $\mathrm{C}_{5} \mathrm{H}_{8}$, where the even/odd weights are $136 / 120=1.13$, for the deuterated form this even/odd ratio is 1.05 , and the effect is too small to be discerned in the intensity variation with $J$ of the unresolved $K^{\prime \prime}=0,1$ features of the $P$-and $R$-branch 
of Figure 4. Similarly, no evidence is seen for any splitting of the $K^{\prime \prime}=2$ and higher $K$ even levels of $\mathrm{C}_{5} \mathrm{D}_{8}$, even though a small $B_{1}, B_{2}$ splitting was detected for the $K^{\prime \prime}=2$ transitions of $\mathrm{C}_{5} \mathrm{H}_{8}[1,2]$.

In the left inset of Figure 4, it is clear that there are extra features in the spectrum, and these are attributed to hot bands that originate from the low frequency $v_{12}\left(b_{1}\right)$ and $v_{25}$ (e) levels, calculated to occur at $236 \mathrm{~cm}^{-1}$ and $264 \mathrm{~cm}^{-1}$, respectively. The anharmonic frequencies calculated for these hot band transitions predict $Q$-branches shifted from $\mathrm{Y}_{5}$ by +0.07 and $-0.27 \mathrm{~cm}^{-1}$, respectively. The latter shift is in reasonable accord with a measured shift of $-0.33 \mathrm{~cm}^{-1}$ for the sharp feature seen in Fig. 4 on the left side of the fundamental $Q$-branch; hence it is assigned as $\left(\mathrm{k}_{5}+\mathrm{v}_{5}\right)-\mathrm{v}_{25}$. A weaker, broader feature also occurs $+0.10 \mathrm{~cm}^{-1}$ above the $\mathrm{u}_{5}$ fundamental, and this is assigned as $\left(\mathrm{v}_{2}+\mathrm{v}_{5}\right)-\mathrm{v}_{2}$.

In addition to added features due to contributions from hot bands, the rovibrational spectrum of $\mathrm{C}_{5} \mathrm{D}_{8}$ is generally condensed compared to that of $\mathrm{C}_{5} \mathrm{H}_{8}$, due to the smaller rotational constants of the former. Moreover, the density of nearby vibrational levels is greater for $\mathrm{C}_{5} \mathrm{D}_{8}$, resulting in a higher probability of Fermi and Coriolis interactions with the upper states of the fundamental transitions. The latter made difficult, for example, the analysis of the apparent $P-Q-R$ structure of the $v_{7}\left(b_{2}\right)$ parallel band predicted near $725 \mathrm{~cm}^{-1}$, since this mode can Coriolis couple to the nearby $\mathrm{M}_{1}\left(b_{1}\right)$ and $v_{3}$ (e) modes predicted at 733 and $719 \mathrm{~cm}^{-1}$, respectively. Evidence for Coriolis perturbation of line positions and intensities were also seen for the intense, overlapping $\mathrm{Y}_{6}\left(b_{2}\right)$ and $\mathrm{k}_{2}(e)$ fundamentals, predicted at 845 and $874 \mathrm{~cm}^{-1}$, respectively. A further complication here is a Fermi resonance interaction with the $v_{8}\left(a_{2}\right)+v_{2}\left(b_{1}\right)$ combination state of $b_{2}$ symmetry which is predicted at $845 \mathrm{~cm}^{-1}$. 
Surprisingly, the $y_{5}\left(b_{2}\right)$ parallel band at $1051 \mathrm{~cm}^{-1}$ proved to be relatively unperturbed, despite the presence of nearby $v_{0}$ and $k_{1}(e)$ fundamentals predicted at higher $\left(1074 \mathrm{~cm}^{-1}\right)$ and lower $\left(1029 \mathrm{~cm}^{-1}\right)$ wavenumber values, respectively. We note that the "push" on the $b_{2}$ rovibrational levels by these two $e$ states is in opposing directions so this compensating effect may account for the fact that over 1600 transitions could be confidently assigned and fitted for $\mathrm{v}_{5}$ without including any Coriolis interactions. This set did not include any of the $Q$-transitions nor the $K^{\prime \prime}=0$ or 1 lines, since none of these were resolved. Despite this good fit, to avoid any possible errors due to unaccounted mixing of the upper levels of the $v_{5}$ transitions, ground state constants were deduced from rotational level separations obtained from $R\left(J^{\prime \prime}-1, K^{\prime \prime}\right)-P\left(J^{\prime \prime}+1, K^{\prime \prime}\right)$ differences in the $v_{5}$ transitions. 458 such differences were obtained and fitted, yielding the three ground state parameters $B_{0}, D_{J}$, and $D_{J K}$ shown in Table 2. We note that these ground state parameters are the same, within the listed uncertainties, as those obtained when these parameters were varied in fitting the full set of the $v_{5}$ transitions. The resultant rovibrational parameters for the upper state of the $v_{15}$ fundamental band are presented in Table 3, and all transitions used in both fits are provided as supplementary data for this article. 


\section{Table 2}

Rotational parameters (in $\mathrm{cm}^{-1}$ ) for the ground state of normal and perdeutero-spiropentane

\begin{tabular}{|c|c|c|c|c|}
\hline \multirow[b]{2}{*}{ Parameter $^{a}$} & \multicolumn{2}{|c|}{$\mathrm{C}_{5} \mathrm{H}_{8}$ [Ref. 1] } & \multicolumn{2}{|c|}{$\mathrm{C}_{5} \mathrm{D}_{8}$} \\
\hline & Experimental & Theoretical $^{\mathrm{b}}$ & Experimental & Theoretical $^{\mathrm{b}}$ \\
\hline$A_{0}$ & {$[0.35059]^{c}$} & 0.35059 & {$[0.24891]$} & 0.24891 \\
\hline$B_{0}$ & $0.1394741(2)$ & 0.13877 & $0.1120700(9)$ & 0.11145 \\
\hline$D_{J} \times 10^{8}$ & $2.462(2)$ & 2.42 & $1.505(27)$ & 1.35 \\
\hline$D_{J K} \times 10^{8}$ & $8.66(4)$ & 8.59 & $3.42(15)$ & 3.51 \\
\hline$D_{K} \times 10^{8}$ & {$[5.21]$} & 5.21 & {$[2.09]$} & 2.09 \\
\hline$H_{J} \times 10^{13}$ & {$[0.0]$} & 0.053 & {$[0.0]$} & 0.029 \\
\hline$H_{J K} \times 10^{13}$ & {$[0.0]$} & -5.45 & {$[0.0]$} & -2.45 \\
\hline$H_{K J} \times 10^{13}$ & {$[0.0]$} & 8.99 & {$[0.0]$} & 4.29 \\
\hline$H_{K} \times 10^{13}$ & {$[0.0]$} & -3.90 & {$[0.0]$} & -1.82 \\
\hline$\Delta_{2} \times 10^{9}$ & $1.172(16)$ & 1.22 & {$[0.0]$} & 0.71 \\
\hline$J_{\max }=$ & 96 & & 52 & \\
\hline$K_{\max }=$ & 31 & & 37 & \\
\hline \# differences & 5612 & & 458 & \\
\hline rms dev. $\left(\mathrm{cm}^{-1}\right)$ & 0.00037 & & 0.00052 & \\
\hline
\end{tabular}

${ }^{a}$ Given in parentheses here and in subsequent tables are uncertainties in the last digits (twice the standard deviation = type $\mathrm{B}, \mathrm{k}=2$, uncertainties as defined in Ref. [16].) ${ }^{\mathrm{b}}$ B3LYP/cc-pVTZ calculation using Gaussian 09 with Anharm/Vibrot option.

${ }^{c}$ Calculated or defined 0.0 values given in square brackets were held fixed. 


\section{Table 3}

$\mathrm{V}_{5}$ rovibrational parameters $\left(\mathrm{cm}^{-1}\right)$ for perdeutero-spiropentane

\begin{tabular}{ccc}
\hline \hline Parameter $^{\mathrm{a}}$ & Experimental & Theoretical $^{\mathrm{b}}$ \\
\hline$\omega$ & $1053.84465(10)$ & 1078.81 \\
$\Delta A \times 10^{3}$ & $-0.2533(6)$ & -0.234 \\
$\Delta B \times 10^{3}$ & $0.1336(3)$ & 0.165 \\
$\Delta D_{J} \times 10^{8}$ & $-0.19(2)$ & \\
$\Delta D_{J K} \times 10^{8}$ & $5.17(6)$ & \\
$\Delta D_{K} \times 10^{8}$ & $-4.13(6)$ & \\
$\Delta H_{J} \times 10^{12}$ & $-0.65(4)$ & \\
$\Delta H_{J K} \times 10^{12}$ & $10.53(18)$ & \\
$\Delta H_{K J} \times 10^{12}$ & $-12.26(24)$ & \\
$\Delta H_{K} \times 10^{12}$ & {$[0]$} & \\
$J_{\max }=$ & 60 & \\
$K_{\max }=$ & 49 & \\
$\#$ transitions & 1633 & \\
$\mathrm{rms} \mathrm{dev.}\left(\mathrm{cm}^{-1}\right)$ & 0.00050 & \\
\hline \hline
\end{tabular}

${ }^{\mathrm{a}} \Delta B=B^{\prime}-B^{\prime \prime}, \Delta C=C^{\prime}-C^{\prime \prime}$, etc.

${ }^{\mathrm{b}}$ B3LYP/cc-pVTZ calculation using Gaussian 09 with Anharm/Vibrot option 


\section{Discussion}

\subsection{Ground state rotational constants and molecular structure}

Table 2 offers comparison of the experimental and theoretical ground state rovibrational constants for normal and perdeutero forms of spiropentane. For both isotopomers, level differences in the ground state were fitted to the symmetric top relation in Equation 3, and it was found that only the constants $B_{0}, D_{J}$, and $D_{J K}$ were needed to obtain excellent fits. No improvement was achieved by including $H$ sextic distortion constants so these were fixed at zero values. Of course, the $K$-dependent $A_{0}$, $D_{K}$, and $H_{K}$ parameters cannot normally be determined from the spectra of symmetric tops but estimates of these parameters can be computed with the Gaussian 09 program [11], and these are also listed in the table. All parameters observed or calculated for $\mathrm{C}_{5} \mathrm{D}_{8}$ are significantly smaller than their $\mathrm{C}_{5} \mathrm{H}_{8}$ counterparts. In the case of the $K^{\prime \prime}=2$ centrifugal splitting constant $2^{\prime \prime}$, the decrease is $42 \%$, and no splitting in any of the $K^{\prime \prime}=2$ transitions was resolved for $\mathrm{C}_{5} \mathrm{D}_{8}$.

The Gaussian calculations of the quartic distortion constants do not contain any anharmonic contribution, and the $D_{J}$ and $D_{J K}$ values correspond to values for the equilibrium structure. The anharmonic corrections are expected to be small however, and the experimental ground state $D_{J}$ and $D_{J K}$ values agree with the theoretical ones in sign and in magnitude to within about $2 \%$, except for $D_{J}$ of $\mathrm{C}_{5} \mathrm{D}_{8}$, where the difference is $12 \%$. The uncertainties in the $\mathrm{C}_{5} \mathrm{D}_{8}$ parameters are much larger than for those for $\mathrm{C}_{5} \mathrm{H}_{8}$, which is not surprising since the latter data set was much larger and included differences from several fundamental bands, with a much greater range of $J$ values.

The $B_{0}$ values from the $\mathrm{C}_{5} \mathrm{H}_{8}$ and $\mathrm{C}_{5} \mathrm{D}_{8}$ analyses are very well-determined, with uncertainties in the seventh decimal place. For both isotopomers, the experimental values 
$0.1394741(1)$ and $0.1120700(9) \mathrm{cm}^{-1}$, respectively, fall between the predicted theoretical values of $B_{e}$ and $B_{0}$ and agree with the latter within $0.6 \%$. We note that the experimental value of $B_{0}$ for $\mathrm{C}_{5} \mathrm{H}_{8}$ is significantly smaller $(1.68 \%)$ than a value of $B_{a}=0.1418 \mathrm{~cm}^{-1}$ calculated from the electron diffraction (ED) thermal average $\left(r_{a}\right)$ structure (given in Table 2 of Ref. 1). Similarly, for $\mathrm{C}_{5} \mathrm{D}_{8}$, the experimental value $0.1120700(9) \mathrm{cm}^{-1}$ is comparably smaller $(1.70 \%)$ than a value of $0.1140 \mathrm{~cm}^{-1}$ calculated from the electron diffraction structure. Since the ED values includes contributions from all thermally populated states, it would be expected that the $B_{a}$ value deduced from the ED structure would actually be lower, not higher, than $B_{0}$. Several authors [17-19] have raised questions about the electron diffraction results, particularly about the $\mathrm{CH}_{2}$ angle, 118.4(9), which is about 4 degrees larger than the Gaussian prediction of 114.4 degrees, and is also larger than a value of 115.0(7) degrees deduced from NMR measurements [18]. As discussed in our earlier paper [1], we suspect a scaling error in the electron diffraction measurements, but this remains to be confirmed.

The $v_{15}$ vibration

Table 3 lists the results for the excited state of $v_{15}$, and it can be seen that the frequency of the band center, $1053.84465(10) \mathrm{cm}^{-1}$ is in good accord $(0.2 \%)$ with the theoretical anharmonic frequency $\left(1051.4 \mathrm{~cm}^{-1}\right)$. The calculated harmonic frequency $\left(1078.8 \mathrm{~cm}^{-1}\right)$ is somewhat higher, indicating that the anharmonicity of this vibrational mode is a modest $2.4 \%$. According to the Gaussian harmonic normal mode calculations, the $v_{15}$ mode is a mixture of ring stretching and $\mathrm{CH}_{2}$ bending motions, with the latter emphasized in the inset of Figure 3, which is a snapshot of the animation of the mode given by the GaussView program. 
The Anharm/Vibrot options of the Gaussian program also permit calculation of the anharmonic contribution to the vibration-rotation constants for each mode $i, \alpha_{4 i}=A_{0}{ }^{\prime \prime}$ $A_{i}{ }^{\prime}=-A_{i}$ and $\alpha_{i}=-B_{i}$. As seen in Table 3, the experimentally-determined $A$ and $B$ constants for the $\mathrm{v}_{5}$ mode of spiropentane agree in sign with the theoretical values but differ in magnitude by $8 \%$ and $-19 \%$, respectively. The $B \%$ difference is somewhat larger than typical values of less than $10 \%$ that we have seen for propellane and bicyclopentane and for other cases where Fermi and/or Coriolis mixing with nearby states is absent. It is likely that unaccounted $\mathrm{x}, \mathrm{y}$ Coriolis mixing of $v_{15}$ with the nearby $v_{0}$ and $V_{21}(e)$ states is responsible, since this can change the $B$ value (but not the $A$ value).

\section{Summary}

This work presents the first infrared and Raman spectra of perdeuterospiropentane, and analysis of the high resolution infrared spectrum of gaseous $\mathrm{C}_{5} \mathrm{D}_{8}$ yielded accurate rovibrational parameters for the ground and excited states of the $v_{15}$ fundamental mode. The $B_{0}$ value determined for the ground state is consistent with our earlier speculation [1] that the structure obtained from electron diffraction measurements is not accurate. Comparison of the experimental and theoretical parameters for the ground state and the upper state levels of the $v_{15}$ parallel band is generally favorable and illustrates the value of quantum calculations as a complement in the analysis of complex molecular spectra. Evidence was seen of significant $l$-doubling and Fermi resonance/Coriolis interactions for some of the other bands of $\mathrm{C}_{5} \mathrm{D}_{8}$, and efforts to analyze these are underway. 
Appendix A. Synthesis of Perdeutero-Spiropentane $\left(C_{5} D_{8}\right)$

Reaction 2 to 3. To a solution of diethyl malonate $(15.3 \mathrm{~mL}, 100.2 \mathrm{mmol})$ in DMF $(20.0 \mathrm{~mL})$ were added potassium carbonate $(34.6 \mathrm{~g}, 250.5 \mathrm{mmol})$, 1,2-dibromoethane- $\mathrm{d}_{4}$ (Alfa Aesar, 99\% D), 2 (25.0 g, 130.3 $\mathrm{mmol})$, and tetrabutyl ammonium bromide $(1.62 \mathrm{~g}, 5.01 \mathrm{mmol})$. The mixture was allowed to stir at room temperature for 12 hours, then was heated to $50^{\circ} \mathrm{C}$ and stirred for another 6 hours. The mixture was diluted with ethyl acetate and was washed with water and brine and dried over $\mathrm{MgSO}_{4}$. The resulting mixture was purified by liquid chromatography (silica gel column), and the solvents were removed under reduced pressure to yield $15.9 \mathrm{~g}$ of deuterated 1,1-dicarbethoxycyclopropane 3 (75.5\%).

Reaction 3 to 4. Diester $3(14.0 \mathrm{~g}, 74.9 \mathrm{mmol})$ was dissolved in $50 \mathrm{~mL}$ ether and added over 1 hour at $0^{\circ} \mathrm{C}$ to a solution of $\mathrm{LiAlD}_{4}(\mathrm{Alfa}, 98 \% \mathrm{D})(4.5 \mathrm{~g}, 98.9 \mathrm{mmol})$ in $200 \mathrm{~mL}$ ether. The reaction was then heated to reflux and allowed to stir for 18 hours. The reaction was quenched by adding sequentially $4.2 \mathrm{~mL}_{2} \mathrm{O}, 4.2$ $\mathrm{mL} 15 \% \mathrm{NaOH}$ solution in $\mathrm{D}_{2} \mathrm{O}$, then $12.6 \mathrm{~mL} \mathrm{D}_{2} \mathrm{O}$. The mixture was filtered and dried over $\mathrm{MgSO}_{4}$. The compound was purified by liquid chromatography, and the solvents removed under reduced pressure to yield $4.96 \mathrm{~g}$ of deuterated 1,1-bis-(hydroxymethy1)cyclopropane 4 (58.9\%).

Reaction 4 to 5. To a solution of $4(4.90 \mathrm{~g}, 48.7 \mathrm{mmol})$ in $95 \mathrm{~mL}$ acetonitrile were added NaI $(21.15 \mathrm{~g}$, $141.1 \mathrm{mmol})$ and tetramethylsilyl chloride $(17.9 \mathrm{~mL}, 141.1 \mathrm{mmol})$ under $\operatorname{Ar}(\mathrm{g})$. The mixture was allowed to stir at room temperature for 20 hours and was diluted with equal volume water and DCM. The organic layer was washed with $10 \% \mathrm{Na}_{2} \mathrm{~S}_{2} \mathrm{O}_{3}$ (aq) and brine and dried over $\mathrm{MgSO}_{4}$. The mixture was filtered and the solvents evaporated under reduced pressure. The crude sample was diluted with hexanes and passed through a silica plug to give $7.48 \mathrm{~g}$ of pure deuterated 1,1-bis-(iodomethy1)cyclopropane $\mathbf{5}(54.8 \%)$.

Reaction 5 to 1 . For convenience, this reaction step employed the same apparatus used in synthesizing ordinary spiropentane from pentaerythrityl tetrabromide, as described in reference 1 . The system consisted of a $500 \mathrm{~mL}$ round bottom flask attached to a water condenser and two successive traps, the first at $-78^{\circ} \mathrm{C}$, the second at $77 \mathrm{~K}$. The system was flushed with $\mathrm{He}(\mathrm{g})$ which was flowed slowly through the system for the entire experiment to carry the product out of the reaction flask. $\mathrm{Na}_{2}$ EDTA $-2 \mathrm{H}_{2} \mathrm{O}(28.52 \mathrm{~g}, 76.6$ mmol), $\mathrm{NaOH}(8.59 \mathrm{~g}, 215 \mathrm{mmol}), \mathrm{NaI}(0.75 \mathrm{~g}, 5.01 \mathrm{mmol}), 150 \mathrm{~mL} \mathrm{H} \mathrm{H}_{2} \mathrm{O}$, and $35 \mathrm{~mL}$ ethanol were added to the flask and the mixture was stirred while heating to $95^{\circ} \mathrm{C}$. $\mathrm{Zn}(\mathrm{s})(6.50,99.4 \mathrm{mmol})$ was then added to the flask and over 1 hour $5(7.48 \mathrm{~g}, 22.6 \mathrm{mmol})$ was added to the stirring solution. The mixture was allowed to stir for 2.5 hours. A small amount of bromine was then added to the spiropentane in the second trap to remove known side products 2-methyl-1-butene and methylene cylobutane. The overall yield of perdeutero-spiropentane 1 was quite low <3\%) but, from infrared spectra, the isotopic purity was high $\sim 90 \%$, and the sample quantity was sufficient for the high resolution spectroscopic measurements. We note that a higher yield $(20 \%)$ in this last reaction step has been reported by House and Lord [12] using Na metal 
to remove the iodine atoms and close the second ring in $\mathbf{1}$, so their method for this last step is recommended.

\section{Acknowledgments}

J. Nibler acknowledges a Camille and Henry Dreyfus Senior Scientist Mentor Award which provided support of B. Erickson. The infrared spectra were recorded at the Pacific Northwest National Laboratory (PNNL) located in Richland, WA, USA. The Pacific Northwest National Laboratory is operated by Battelle Memorial Institute for the U.S. Department of Energy under Contract No. DE-AC05-76RL01830.

\section{References}

1. J.E. Price, K.A. Coulterpark, T. Masiello, J.W. Nibler, A. Weber, A. Maki, T.A. Blake, J. Mol. Spec. 269 (2011) 129-136.

2. A. Maki, J.E. Price, J. Harzan, J.W. Nibler, A. Weber, T. Masiello, T.A. Blake, J. Mol. Spec. 312 (2015) 68-77.

3. R. Kirkpatrick, T. Masiello, N. Jariyasopit, A. Weber, J.W. Nibler, A. Maki, T.A. Blake, T. Hubler, J. Mol. Spectrosc. 248 (2008) 153-160.

4. R. Kirkpatrick, T. Masiello, N. Yariyasopit, J.W. Nibler, A. Maki, T.A. Blake, A. Weber, J. Mol. Spectrosc. 253 (2009) 41-50.

5. A. Maki, A. Weber, J.W. Nibler, T. Masiello, T.A.Blake, R. Kirkpatrick. J. Mol. Spectrosc. 264 (2010) 26-36.

6. M.A. Martin, A. Perry, T. Masiello, K.D. Schwartz, A. Weber, A. Maki, T.A. Blake, J. Mol. Spectrosc. 262 (2010) 42-48 
7. A. Perry, M.A. Martin, J.W. Nibler, A. Maki, A. Weber, T.A. Blake, J. Mol. Spectrosc. 276 (2012) 22-32.

8. D.E. Applequist, G.F. Fanta, B.W. Henrikson, J. Org. Chem. 23 (1958) 17151716.

9. K.R. Mondanaro, W.P. Dailey, Organic Syntheses, Coll. Vol. 10 (2004) 658 (2004); Vol. 75 (1998) 98.

10. K.B. Wiberg, S.T. Waddell, J. Am. Chem. Soc. 112 (1990) 2194-2216.

11. Gaussian 09W, Revision B.01, M.J. Frisch, G.W. Trucks, H.B. Schlegel, G.E. Scuseria, M.A. Robb, J.R. Cheeseman, G. Scalmani, V. Barone, B. Mennucci, G. A. Petersson, H. Nakatsuji, M. Caricato, X. Li, H.P. Hratchian, A.F. Izmaylov, J. Bloino, G. Zheng, J.L. Sonnenberg, M. Hada, M. Ehara, K. Toyota, R. Fukuda, J. Hasegawa, M. Ishida, T. Nakajima, Y. Honda, O. Kitao, H. Nakai, T. Vreven, J.A. Montgomery, Jr., J.E. Peralta, F. Ogliaro, M. Bearpark, J.J. Heyd, E. Brothers, K.N. Kudin, V.N. Staroverov, T.Keith, R. Kobayashi, J. Normand, K. Raghavachari, A. Rendell, J.C. Burant, S.S. Iyengar, J. Tomasi, M. Cossi, N. Rega, J.M. Millam, M. Klene, J.E. Knox, J.B. Cross, V. Bakken, C. Adamo, J. Jaramillo, R. Gomperts, R. E. Stratmann, O. Yazyev, A. J. Austin, R. Cammi, C. Pomelli, J. W. Ochterski, R.L. Martin, K. Morokuma, V.G. Zakrzewski, G.A. Voth, P. Salvador, J.J. Dannenberg, S. Dapprich, A.D. Daniels, O. Farkas, J.B. Foresman, J.V. Ortiz, J. Cioslowski, D.J. Fox, Gaussian, Inc., Wallingford CT, 2010.

12. H.O. House, R.C. Lord, H.S. Rao, J. Org. Chem., 21 (1956) 1487-1491.

13. G. Dallinga, R.K. van der Raai, L.H. Toneman, Rec. Trav. Chim. Pays-Bas 87 (1968) 897-905.

14. A. Weber, J. Chem. Phys. 73 (1980) 3952-3972; 74 (1981) 4754.

15. G. Herzberg, Electronic Spectra of Polyatomic Molecules,. p. 224, D. van Nostrand, New York (1966).

16. B.N. Taylor, C.E. Kuyatt, NIST Technical Note No. 1297 (1994), Guidelines for Evaluating and Expressing the Uncertainty of NIST Measurement Results. This publication may be downloaded from $<$ http://physics.nist.gov/Pubs/ guidelines/contents.html>

17. A.D. Buckingham, E.E. Burnell, C.A. deLande, Mol. Phys. 17 (1969) 205-207.

18. W. Bechtold, J.H. Goldstein, J. Am. Chem. Soc. 103 (1981) 4989-4991.

19. H. Dodziuk, J. Leszczynski, K. Jackowski, Tetrahedron 57 (2001) 5509-5513. 


\section{List of Tables}

1. Experimental and calculated fundamental frequencies $\left(\mathrm{cm}^{-1}\right)$ of perdeuterospiropentane.

2. Rotational parameters (in $\mathrm{cm}^{-1}$ ) for the ground state of normal and perdeuterospiropentane

3. $\quad \mathrm{M}_{5}$ rovibrational parameters $\left(\mathrm{cm}^{-1}\right)$ for perdeutero-spiropentane.

\section{List of Figures}

1. Reaction sequence for the synthesis of perdeutero-spiropentane $\left(\mathrm{C}_{5} \mathrm{D}_{8}\right)(\mathrm{V})$.

2. Infrared and Raman spectra of perdeutero-spiropentane $\left(\mathrm{C}_{5} \mathrm{D}_{8}\right)$. For display purposes, intense features due to $\mathrm{CO}_{2}$ and $\mathrm{H}_{2} \mathrm{O}$ were deleted from the spectra. Assignments of most of the fundamental modes are indicated in the spectrum. Vertical sticks indicate the positions calculated at the anharmonic level using Gaussian (B3LYP/cc-pVTZ), with stick heights representing relative intensities computed at the harmonic level.

3. Diagram for the allowed transitions between $A_{1}$ and $B_{2}$ vibrational states for the $\mathrm{V}_{5}$ band of perdeutero-spiropentane, $\mathrm{C}_{5} \mathrm{D}_{8}$.

4. $\quad y_{5}\left(b_{2}\right)$ parallel band of perdeutero-spiropentane. The inset to the left shows the $K^{\prime \prime}$-structure of the $J^{\prime \prime}=20$ and $19 P$-lines. Some of the extra features, as well as the sharp $Q$-feature on the left side of the $Q$-branch are attributed to hot bands, such as $\left(v_{5}+v_{5}\right)-v_{25}$. The rising baseline in the $R$-branch is due to overlap with a nearby $v_{0}(e)$ perpendicular band at $1081 \mathrm{~cm}^{-1}$. 